\title{
Yeast telomerase and the SUN domain protein Mps3 anchor telomeres and repress subtelomeric recombination
}

\author{
Heiko Schober, ${ }^{1,2}$ Helder Ferreira, ${ }^{2}$ Véronique Kalck, ${ }^{2}$ Lutz R. Gehlen, ${ }^{2}$ and Susan M. Gasser ${ }^{1,2,3}$ \\ ${ }^{1}$ NCCR Frontiers in Genetics, University of Geneva, 1211 Geneva, Switzerland; ${ }^{2}$ Friedrich Miescher Institute \\ for Biomedical Research, 4058 Basel, Switzerland
}

Telomeres form the ends of linear chromosomes and protect these ends from being recognized as DNA doublestrand breaks. Telomeric sequences are maintained in most cells by telomerase, a reverse transcriptase that adds TG-rich repeats to chromosome ends. In budding yeast, telomeres are organized in clusters at the nuclear periphery by interactions that depend on components of silent chromatin and the telomerase-binding factor yeast $\mathrm{Ku}(\mathrm{yKu})$. In this study, we examined whether the subnuclear localization of telomeres affects end maintenance. A telomere anchoring pathway involving the catalytic yeast telomerase subunits Est2, Est1, and Tlc1 is shown to be necessary for the perinuclear anchoring activity of Yku80 during $\mathrm{S}$ phase. Additionally, we identify the conserved Sad1-UNC-84 (SUN) domain protein Mps3 as the principal membrane anchor for this pathway. Impaired interference with Mps3 anchoring through overexpression of the Mps3 $\mathrm{N}$ terminus in a tel1 deletion background led to a senescence phenotype and to deleterious levels of subtelomeric $\mathrm{Y}^{\prime}$ recombination. This suggests that telomere binding to the nuclear envelope helps protect telomeric repeats from recombination. Our results provide an example of a specialized structure that requires proper spatiotemporal localization to fulfill its biological role, and identifies a novel pathway of telomere protection.

[Keywords: Telomere protection; telomerase; nuclear envelope; Sad1/UNC-84 (SUN) homology domain; Mps3; nuclear organization; ATM homolog Tel1]

Supplemental material is available at http://www.genesdev.org.

Received February 3, 2009; revised version accepted March 17, 2009.

The sequestration of different types of chromatin into distinct subnuclear compartments is thought to help regulate nuclear processes such as gene activation, silencing, and DNA repair (for review, see Sexton et al. 2007). A well-studied example is provided by the clustering of the 32 budding yeast telomeres into two to eight foci that are enriched at the nuclear periphery. This localization is mediated by two redundant pathways that require silent information regulatory 4 (Sir4), a silencing factor, and the yeast $\mathrm{Ku}(\mathrm{yKu})$ heterodimer (Hediger et al. 2002; Taddei et al. 2004). Sir4 anchors repressed chromatin to the nuclear envelope (NE) through its partitioning and anchoring domain (PAD, residues 950-1262). This domain specifically binds Esc1 (Enhancer of silent chromatin 1), an acidic protein of low abundance that is associated exclusively with the inner face of the NE (Andrulis et al. 2002; Gartenberg et al. 2004; Taddei et al. 2004). Esc1 is excluded from nuclear pores but localizes independently of Sir4 in patches along the

${ }^{3}$ Corresponding author.

E-MAIL Susan.Gasser@fmi.ch; FAX 41-61-697-3976.

Article is online at http://www.genesdev.org/cgi/doi/10.1101/gad.1787509. nuclear membrane (Taddei et al. 2004). Although Yku80 and Sir4 interact, Yku80 is able to relocate chromatin to the NE in the absence of Sir4 and Esc1 (Taddei et al. 2004), indicating that it binds another, as yet unidentified, membrane-bound factor. Apart from its roles in telomere anchoring, capping, and silencing, the yKu heterodimer also acts as a positive regulator of telomerase by direct interaction with Tlc1, the RNA subunit of telomerase (Stellwagen et al. 2003; Ting et al. 2005).

Telomerase is a reverse transcriptase conserved in nearly all eukaryotes. It is necessary for the maintenance of their genomes, which are propagated as linear chromosomes. Chromosomal ends, or telomeres, shorten progressively during successive rounds of replication and cell division. Telomerase counteracts this so-called "end replication problem" by templating DNA synthesis from telomerase RNA (Lingner et al. 1995).

Mutations in telomerase lead to chromosomal rearrangements due to aberrant end-joining events, which occur frequently in the early stages of cancer. In higher eukaryotes, the colocalization of telomerase components to subnuclear Cajal bodies has been shown to play a role in regulating its activity (Jady et al. 2006; Tomlinson 
et al. 2006; Cristofari et al. 2007; Venteicher et al. 2009). In addition, there is evidence that the binding of budding yeast subtelomeric factors, which antagonize telomere association with the NE, negatively affects telomerase efficiency (Berthiau et al. 2006; Hediger et al. 2006).

A recent study has suggested that the integral nuclear membrane protein Mps3, the sole Saccharomyces cerevisiae member of a conserved family of proteins bearing a conserved Sad1-UNC-84 (SUN) homology domain, contributes to telomere anchoring (Bupp et al. 2007). In higher eukaryotes, SUN domain proteins bridge inner and outer membranes of the NE, interacting with both the cytoskeleton and nucleoskeleton (Tzur et al. 2006). The Schizosaccharomyces pombe homolog Sad1 contributes to telomere clustering in meiosis through interactions with two additional proteins, Bqt1 and Bqt2 (Chikashige et al. 2006). The budding yeast homolog Mps3 spans the inner NE once and plays a major role in spindle pole body (SPB) organization (Jaspersen et al. 2002; Nishikawa et al. 2003). Recently, it was also shown to play a role in the Esc1-independent anchoring of telomeres in S-phase cells (Bupp et al. 2007).

As mentioned above, Yku80 is able to anchor chromatin and telomeres to the nuclear periphery in the absence of Esc1 (Gartenberg et al. 2004; Taddei et al. 2004). We therefore set out to identify the peripheral partner involved in Yku80 anchorage. This also allowed us to explore the role of nuclear compartmentation in the protection and maintenance of telomeric structures, through the use of live-cell imaging and reverse genetic tools. This led to the discovery that the interaction between $\mathrm{yKu}$ and telomerase is required for Yku80 anchoring at the NE in S-phase cells.

We found that the telomerase core subunit Est2 itself can relocate chromatin to the $\mathrm{NE}$ in a manner dependent on Est1 and Mps3. Mps3 is also needed for yKu-mediated chromatin anchoring in S-phase cells, and neither Yku80 nor Est2 tethers chromatin to nuclear pores. Finally, we show that the overexpression of a soluble $\mathrm{N}$-terminal domain of Mps3 relieves telomere anchoring in S phase. Overexpression of this Mps3-N domain in strains lacking the ATM-kinase homolog Tell further led to a senescencelike phenotype and to unprotected, hyperrecombinant telomeres. This argues strongly that anchorage through Mps3 and telomere helps suppress recombination at telomeric repeats.

\section{Results}

Yku80-Tlc1 interaction is critical for perinuclear anchoring by targeted Yku80

The yKu heterodimer has been shown to interact directly with a stem-loop of the telomerase RNA, Tlc1 (Stellwagen et al. 2003). Given that yKu can anchor telomeres independently of Sir4 (Hediger et al. 2002), we examined whether the Yku80-Tlc1 interaction might contribute to telomere positioning. To address this, we exploited a yku 80 mutant that has lost the ability to bind Sir4 (yku80-4) (Roy et al. 2004; Taddei et al. 2004), and fused this mutant allele in-frame to a sequence encoding the bacterial DNA-binding factor lexA. When bound to a lacO/lexA-tagged chromosomal locus (ARS607), the lexA-yku80-4 fusion shifted ARS607 from a random to a peripheral position in both G1- and S-phase cells (Fig. 1C).

To see if this relocalization required the interaction of Yku80 with telomerase, we performed the same experiment in a strain bearing a mutation in TLC1 that abolishes the interaction between YKu80 and telomerase RNA (tlc $\Delta 48$ ) (Fig. 1A; Stellwagen et al. 2003). In this background, the binding of lexA-yku80-4 failed to shift the tagged locus to the nuclear periphery in S-phase cells (Fig. 1C). Intriguingly, the loss of anchoring was much less pronounced in G1 phase. Although it is unclear how cell cycle stage affects localization, this result confirms previously reported differences between G1- and S-phase anchoring by Yku80 (Taddei et al. 2004).

To confirm that the S-phase-specific loss of anchoring indeed reflects the loss of Yku80-Tlc1 interaction, we tested the reciprocal mutation in Yku80, which impairs its recognition of the Tlc1 stem-loop structure (yku80135i) (Fig. 1A). Again, relocalization activity was lost in S-phase cells (Fig. 1D). Combining the "135i" and "4" mutations into one yku80 allele such that the lexA-yKu fusion binds neither Tlc1 nor Sir4 (lexA-yku80-4-135i) (Fig. 1A) completely abrogated the affinity of Yku80 for the NE, again in S-phase cells (Fig. 1D). We conclude that, in S phase, Yku80 anchoring to the NE is dependent on its ability to bind the telomerase RNA Tlc1.

\section{The telomerase catalytic core subunit Est2 localizes} chromatin to the NE

The dependence of Yku80 on Tlcl for peripheral targeting prompted us to test whether the catalytic core component of telomerase Est2 also targets chromatin to the NE. To eliminate senescence phenotypes that occur upon est2 deletion, we made use of the relocalization assay, this time using a lexA-Est2 fusion targeted to the lacOtagged ARS607 locus. We found that lexA-Est2 was able to recruit chromatin to the nuclear periphery in both G1and S-phase cells, whereas lexA alone could not (Fig. 2A).

We next asked whether Est1, an accessory component of telomerase, is involved in the Est2 anchoring pathway. We repeated the relocalization assay for lacO-tagged ARS607 in strains lacking Est1 (Fig. 2B). Since est1 deletion causes a senescence phenotype, these experiments were done on cultures derived from multiple est $1 \Delta$ spores grown up immediately after tetrad dissection. We found that yku80-4 as well as Est2 fail to anchor in S phase when Est1 is not present.

These results suggested that telomerase, specifically the Est1 subunit, has a binding partner at the NE. This binding partner is not Esc1, or at least not exclusively Esc1, since deletion of esc1 led to an insignificant drop in relocalization efficiency (see $P$-values) (Fig. 2A). We note that both Yku80 and Est2 can target DNA efficiently to the NE in both G1- and S-phase cells, while Yku80-mediated relocation depends on Tlc1 and Est1 only in S phase. This may indicate either that yKu binds 


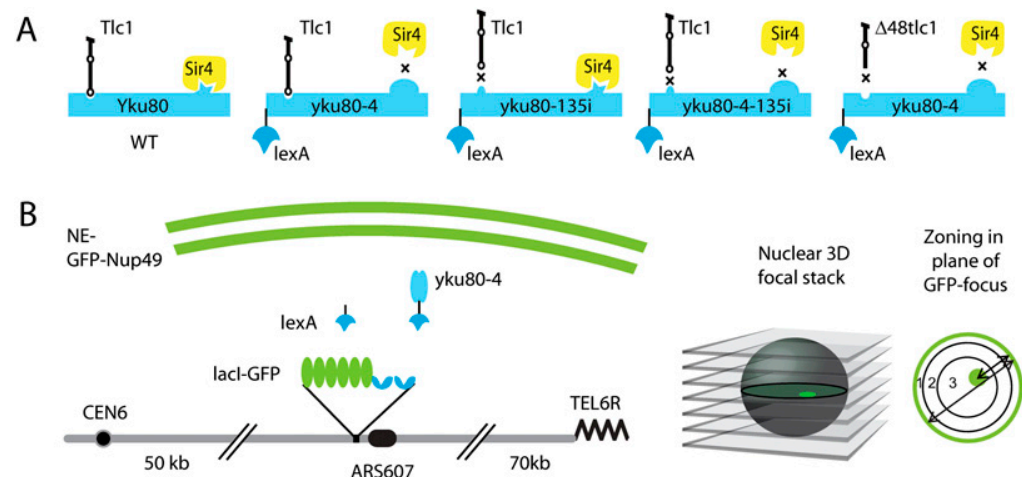

Figure 1. Yku80-mediated $\mathrm{NE}$ anchoring in $\mathrm{S}$ phase requires interaction with Tlc1. (A) Scheme of different Yku80 proteins and their interaction partners relevant for this study. (B) We scored the efficiency of DNA position by taking threedimensional (3D) through-focal stacks of images of living yeast cells and scoring the position of the GFP-tagged locus with respect to GFP-Nup49 in the focal plane of the GFP focus. Relative positions from $>100$ cells were binned into three zones, and deviations from a random distribution were scored for statistical significance (Hediger et al. 2004). $(C, D)$ Zoning assay of GA-1461 (wild type) transformed with (+) lexA alone (G1, $n=158 ; \mathrm{S}, n=87)$; GA-1461 (wild type) + lexA-yku80-4 (G1, $n=147$; S $n=113) ;$ and GA-2814 (tlc1 $\Delta 48)+$ lexA-yku80-4 (G1, $n=255$; S, $n=170)(C)$, and GA-1461 (wild type) + lexA-yku80-4 (G1, $n=147$; S, $n=113$ ); GA1461 (wild type) + lexA-yku80-135i (G1, $n=215$; S, $n=169$ ); and GA-1461 (wild type) + lexA-yku80135i-4 (G1, $n=195 ; \mathrm{S}, n=129)(D)$.
C
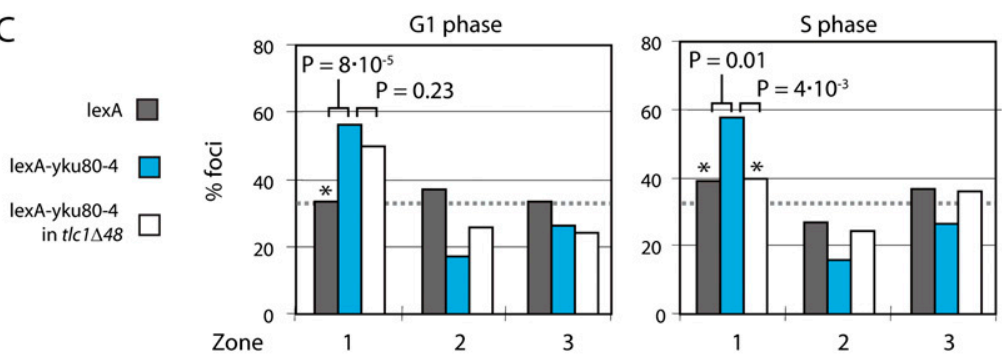

D

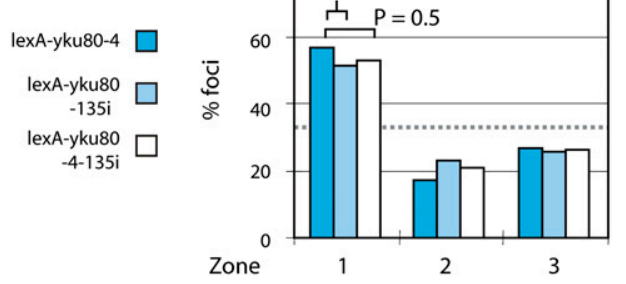

Est2 independently of Tlc1 in G1, or that yKu binds another as yet unidentified perinuclear protein in G1.

\section{yKu80 and Est2 do not recruit chromatin to the nuclear pore complex (NPC)}

We speculated that the perinuclear anchor of Yku80 and telomerase might be a component of the NPC. The deletion of genes encoding nuclear pore proteins (Nup) is often pleiotropic, since they affect nuclear macromolecular transport (Zhao et al. 2004; Loeillet et al. 2005; Palancade et al. 2007). For this reason, relocation assays in strains deleted for Nup genes are rarely conclusive. Instead, we made use of a strain carrying a small N-terminal deletion of NUP133, which shifts all pores into one large cluster while maintaining normal poly $(\mathrm{A})^{+}$RNA export (Doye et al. 1994). We used CFP-Nup49 to visualize the position of the pore cluster and inserted an array of lacO- and lexA-binding sites near LYS2, a gene found in the middle of the right arm of chromosome 2 (Chr 2) (Fig. 2C). Expression of lacI-GFP and various lexA fusion constructs allowed us to determine whether or not the tagged LYS2 locus was recruited to the NPC. As a positive control for pore association, we expressed lexA-Nup84, an integral NPC component.
We found that the expression of lexA-Nup84 doubled the frequency with which the tagged LYS2 locus coincided with CFP-Nup49 in S-phase cells (i.e., coincidence of $42 \%$ vs. $19 \%$ in the presence of lexA alone). The expression of lexA-yku80-4 or lexA-Est2, on the other hand, gave but a slight and statistically insignificant increase in LYS2 pore coincidence over lexA (Fig. 2D). lexA-yku80-4, lexA-Est2, and lexA-Nup84 all shift DNA to the nuclear periphery in $\sim 60 \%$ of the cells scored (Figs. 1, 2A; Nagai et al. 2008), thus the 25\% coincidence with CFP-Nup49 makes it unlikely that the perinuclear anchor of Yku80 and telomerase is an integral NPC component.

In order to evaluate the significance of these values, we calculated the degree of colocalization of a randomly distributed spot with a pore cluster. Simulation of random colocalization of a focus of $250 \mathrm{~nm}$ in diameter within a pore cluster shows that the frequency of chance colocalization depends on the cluster length and width, and on the fraction of foci found in zone 1 (Supplemental Fig. S1). After we eliminated the nucleolar volume from the potential nuclear space occupied by LYS2, computer simulation indicated that a randomly distributed tagged locus would coincide with a pore cluster in $9 \%$ of the cells (pore cluster dimension set to $800 \mathrm{~nm} \times 340 \mathrm{~nm}$ ). A 
A

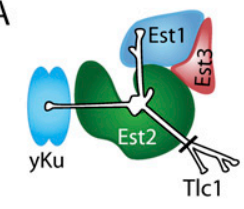

lexA-Est2

lexA-Est2 in esc1A
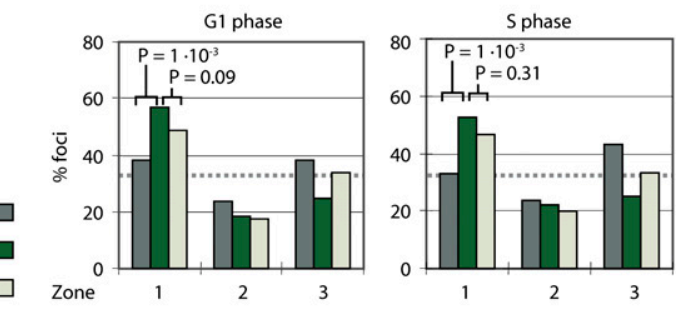

B
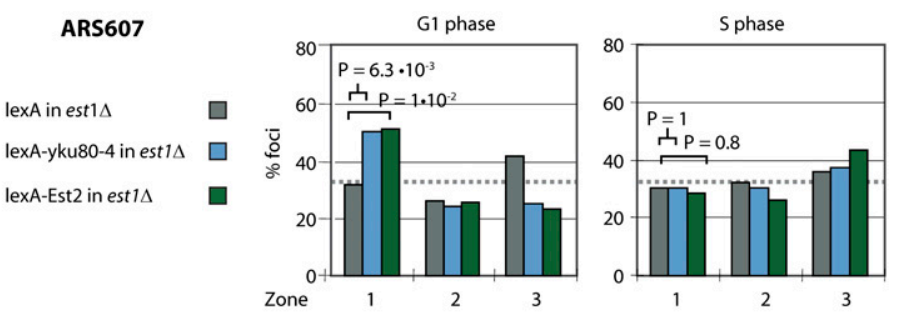

C
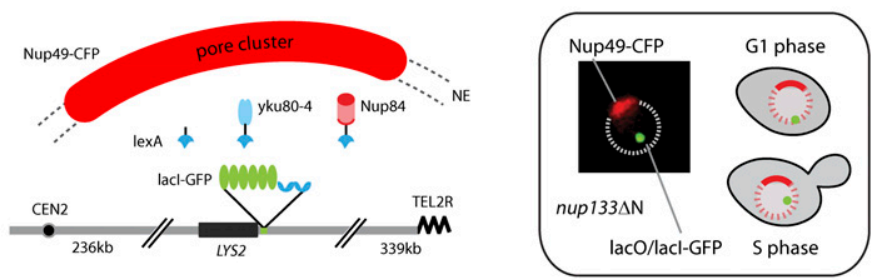

D
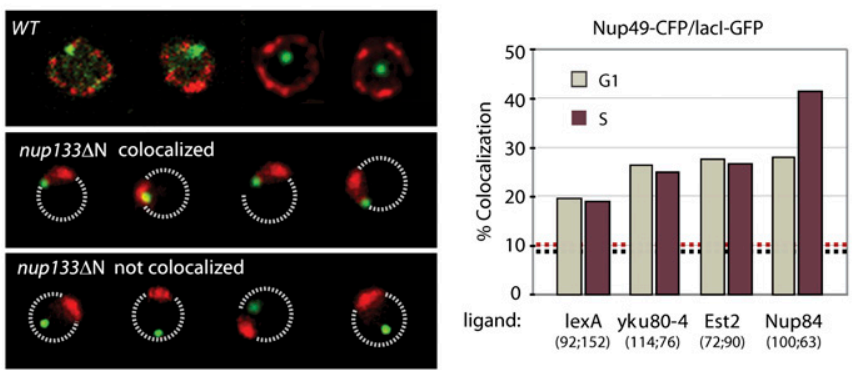

Figure 2. Est2 relocates a randomly distributed locus to the NE in an Est1-dependent manner. $(A, B)$ The targeted anchoring assay for ARS607 was performed as described in Figure 1B. (A) Strains and numbers of cells counted are as follows: GA-1461 (wild type) + lexA alone (G1, $n=131$; $\mathrm{S}, n=118$ ), GA-1461 (wild type) + lexA-Est2 (G1, $n=190$; $\mathrm{S}, n=167)$, and GA-1950 (esc1 1$)+$ lexA-Est $2(\mathrm{G} 1, n=269$; $S, n=120)$. $P$-values for wild type + lexA vs. esc $1 \Delta+$ lexAEst2: $\mathrm{G} 1$ phase, $P=0.047$; $\mathrm{S}$ phase, $P=0.032$. $(B)$ Haploid yeast were sporulated from diploid GA-5559 (est1 $\Delta$ ) previously transformed with lexA (G1 phase, $n=69$; S phase, $n=52$ ); lexA-yku80-4 (G1 phase, $n=252$; $\mathrm{S}$ phase, $n=126$ ), or lexA-Est2 (G1 phase, $n=121$; $S$ phase, $n=86$ ). $(C)$

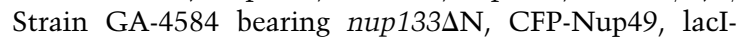
GFP, and lexA-lacO integrated at LYS2 (green spot) was used. In this strain, pores cluster at one site of the NE and are visualized by CFP-Nup49 (red). (D) Either lexA, lexAyku80-4, lexA-Est2, or lexA-Nup84 fusion proteins were expressed and scored for their ability to shift the lacOlexA-tagged LYS2 locus to the CFP-Nup49 cluster. Tangency and overlap were both considered as colocalization. Cell cycle was scored based on the presence of buds $(\mathrm{S}$ phase). The numbers of nuclei scored for each condition are indicated. The bottom dashed line indicates expected colocalization for a randomly distributed locus $(9 \%)$, and the top dashed line indicates expected colocalization of a peripherally enriched locus $(60 \%$ zone $1 ; 9.9 \%)$ (Supplemental Fig. S1) A proportional analysis test with a Bonferroni multiple test correction showed that only lexA-Nup84 significantly colocalizes with pores. lexA versus lexA-Nup84 (S phase), $P=0.002$; lexA versus lexA-yku80-4 (S phase), $P=0.9$; lexA versus lexA-Est2 (S phase), $P=0.85$. locus that is in zone $160 \%$ of the time would coincide with a pore cluster in $10 \%$ of cases (Supplemental Fig. S1). Since the binding of lexA, which has no physiological interaction with the NPC, yielded a $20 \%$ level of colocalization, we suspect that some element or gene in the vicinity of LYS2 may have affinity for the NPC. On the other hand, the zone 1 values of $24 \%-27 \%$ scored for lexA-yku80-4 and lexA-Est2 are not significantly higher than those obtained with lexA alone (Fig. 2D). This argues that neither Yku80 nor Est2 associates significantly with the NPC. Consistent with this finding, we found that double deletion of two NPC-associated proteins (Mlp1 and Mlp2), which were once proposed to bind $\mathrm{yKu}$, do not affect the efficiency with which Yku80 or yku80-4 shift ARS607 to the NE (Supplemental Fig. S2).

\section{Telomerase-mediated recruitment to the NE depends on Mps3}

The conserved budding yeast SUN domain protein Mps3 is an integral inner nuclear membrane protein whose
N-terminal domain faces the nuclear interior (Fig. 3A). Mps3 was shown to bind Est1 by in vitro immunoprecipitation and yeast two-hybrid assays (Antoniacci et al. 2007). It was shown recently that Mps3 also interacts with a domain upstream of the Sir4 PAD domain, and that Mps3 is needed for proper telomere anchoring in S-phase cells (Bupp et al. 2007).

To test whether Mps3 is the peripheral anchor for telomerase, we made use of the targeted anchoring assay again, but expressed lexA-Est2 and lexA-yku80-4 in cells bearing a deletion of amino acids 75-150 of Mps3, which eliminates the nucleoplasmic anchorage site for telomeres (see Bupp et al. 2007). Using this deletion background and an isogenic parental strain that expresses an intact Mps3, we found that both lexA-Est2 and lexAyku80-4 require this nucleoplasmic domain of Mps3 to shift a tagged internal locus to the NE (Fig. 3B,C). The statistical significance and robustness of this dependence is striking. This argues that an anchoring pathway that involves $\mathrm{yKu}$, telomerase, and the nuclear membrane protein Mps3 functions in both G1- and S-phase cells, 
A

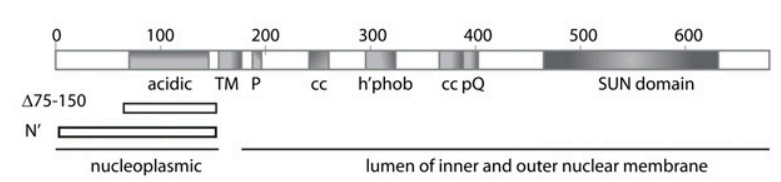

B

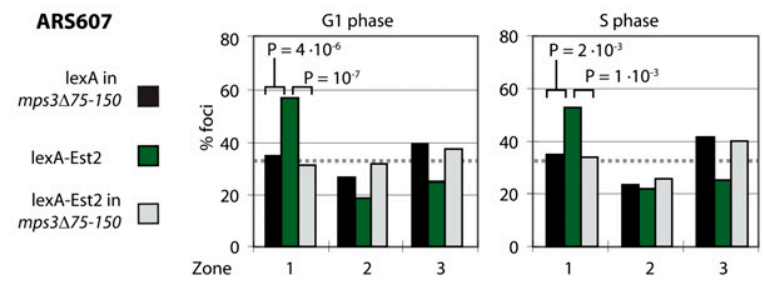

C
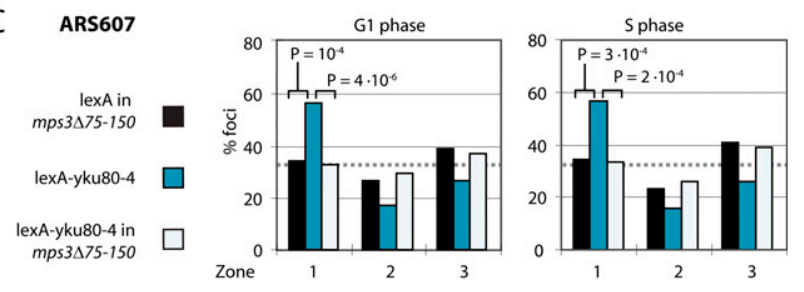

Figure 3. Mps3 $\mathrm{N}$ terminus is required for $\mathrm{Yku} 80$ and Est2 anchoring. (A) Domain organization of Mps3 shows the acidic region, transmembrane domain (TM), a potential Walker P-loop $(\mathrm{P})$, two coiled-coil regions (cc), a hydrophobic region (h'phob), a polyglutamine stretch (pQ), and a SUN domain (see Conrad et al. 2007). The deletion amino acids $75-150$ and overexpressed $\mathrm{N}^{\prime}$ domain (amino acids 1-153) are indicated. (B) GA-5175 $(m p s 3 \Delta 75-150)+$ lexA (G1 phase, $n=151$; S phase, $n=140)$, GA-1461 (wild type) + lexA-Est2 (G1 phase, $n=190$; $\mathrm{S}$ phase, $n=$ 167), and GA-5175 (mps3s75-150) + lexA-Est2 (G1 phase, $n=$

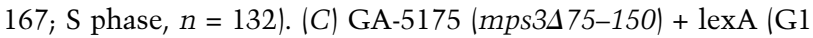
phase, $n=151$; $S$ phase, $n=140$ ), GA-1461 (wild type) + lexAku80-4 (G1 phase, $n=147$; $S$ phase, $n=113$ ), and GA-5175 $(m p s 3 \Delta 75-150)+$ lexA-yku80-4 (G1 phase, $n=151$; S phase, $n=$ $140)$.

although its dependence on Yku80-Tlc1-Est1 interaction is cell cycle-controlled and S-phase-specific. We speculate that in S phase yKu exploits telomerase to bind Mps3, whereas in G1 phase, when a cell cycle-dependent degradation depletes Est1 mRNA (Larose et al. 2007), yKu either binds Est2 directly or interacts with another, unidentified peripheral protein. We note that Est2 seems to be able to interact with Mps3 throughout the cell cycle, thus control over this interaction may be imposed by a modification of yKu.

\section{Ectopic expression of the Mps3 $N$ terminus can disrupt artificial tethering}

To demonstrate that the loss of anchoring does not stem from indirect effects, we expressed a dominant-negative allele of MPS3 that contains only its nucleoplasmic $\mathrm{N}$-terminal domain fused to tetR-mCherry. This fusion stabilized the domain and allowed us to monitor its expression (Shaner et al. 2004). Unlike the perinuclear full-length Mps3 protein (Bupp et al. 2007), Mps3$\mathrm{N}^{\prime}$-tetR-mCherry $\left(\mathrm{Mps} 3-\mathrm{N}^{\prime}\right)$ diffused freely throughout the nucleoplasm (see below). We then performed the relocalization assay using a lacO-LexA array inserted at ARS514, found in the middle of Chr5R (Fig. 4A), to make sure that anchoring effects were not locus-specific. We found that the overexpression of $\mathrm{Mps} 3-\mathrm{N}^{\prime}$ impaired the ability of both lexA-Est2 (Fig. 4B) and lexA-yku80-4 (Fig. 4C) to recruit the tagged locus to the NE. As expected, Mps3-N' had no effect on anchoring mediated by a transmembrane protein, Yif1. That is, lexA-Yif1 shifted ARS514 to the nuclear periphery with or without Mps3$\mathrm{N}^{\prime}$ coexpression (Fig. 4D). We conclude from these lossof-function and dominant-negative studies that the pathway of NE anchorage involving Yku80 and telomerase is

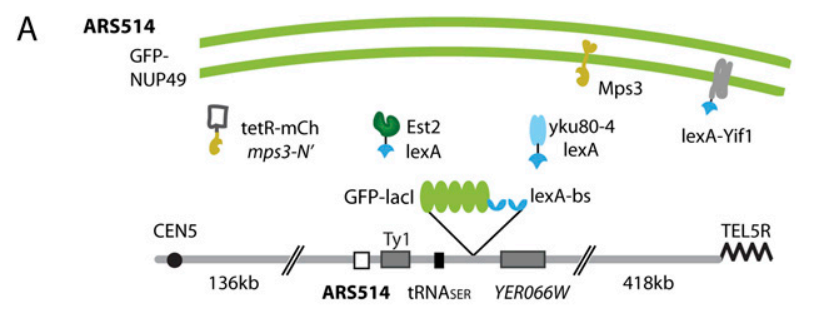

B
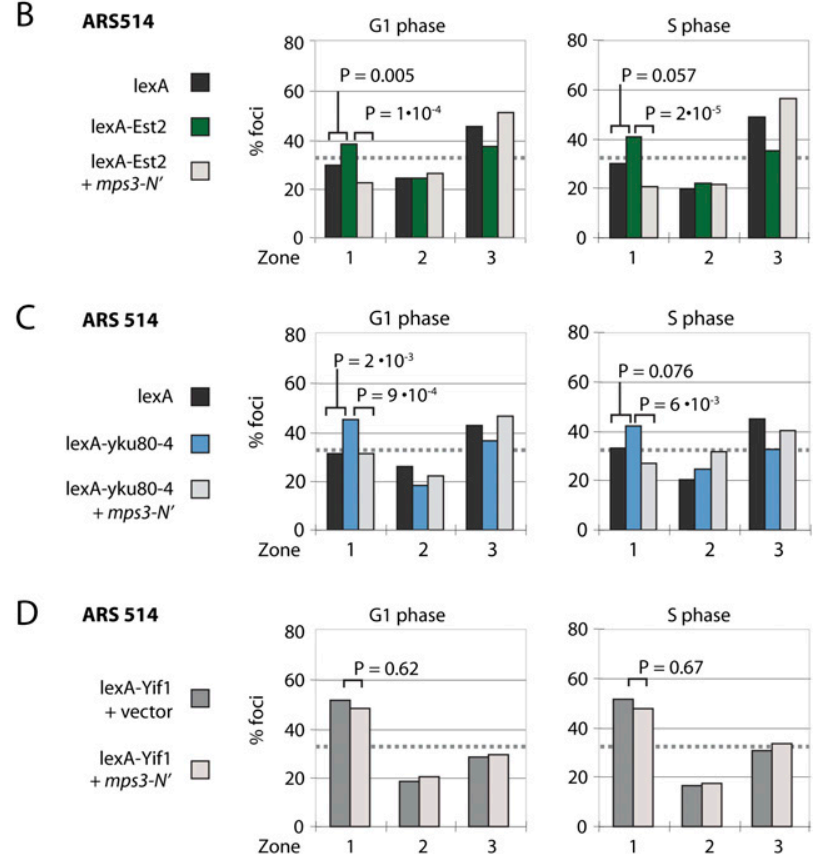

Figure 4. Mps3- $\mathrm{N}^{\prime}$ expression antagonizes the perinuclear anchoring of ARS514 by Est 2 and Yku80. (A) Scheme of targeted anchoring for lacO repeats integrated in the middle of Chr5R near ARS514 for the relocation assay. (B) The position of ARS514 was scored as in Figure 1B with the following strains and ligands: GA-3690 (wild type) + lexA (G1 phase, $n=604$; S phase, $n=444$ ), GA-3690 (wild type) + lexA-Est2 (G1 phase, $n=$ 374; S phase, $n=204$ ), and GA-3690 (wild type) + lexA-Est $2+$ Mps3-N' (G1 phase, $n=282 ; \mathrm{S}$ phase, $n=170$ ). (C) GA-3690 (wild type) + lexA (G1, $n=239 ;$ S, $n=221$ ), GA-3690 (wild type) + lexA-yku80-4 (G1, $n=228 ; \mathrm{S}, n=158$ ), and GA-3690 (wild type) + lexA-yku80-4 + Mps3-N' (G1, $n=317$; S, $n=150)$. (D) GA-3690 (wild type) + lexA-Yif1 (G1 phase, $n=86$; $S$ phase, $n=42$ ), and GA-3690 (wild type) + lexA-Yif1 + Mps3-N' (G1 phase, $n=153$; S phase, $n=91$ ). 
indeed mediated by interaction with the Mps3 N-terminal domain.

\section{Telomere anchoring requires the inner nuclear membrane protein Mps3 in S-phase cells}

We next asked whether the results obtained in these "targeted relocalization" assays accurately reflect the behavior of a native telomere in a wild-type background. Although Bupp et al. (2007) scored a significant reduction in telomere anchoring in the mps3s75-150 strain, indirect side effects might have arisen from the abrogation of SPB function. We sought to counter this criticism by titrating out telomere-binding sites for the intact Mps3 with a soluble, overexpressed $\mathrm{N}$-terminal domain of Mps3. Indeed, expression of $\mathrm{Mps} 3-\mathrm{N}^{\prime}$ in a yeast strain that contains a fluorescently tagged telomere 6R (Tel6R) showed a strong reduction in its perinuclear anchoring in S-phase cells (Fig. 5A). Our results are consistent with those obtained by Bupp et al. (2007), who observed Mps3dependent association of Tel6R to the NE in S-phase cells only. This result is particularly relevant because Tel6R anchoring is known to be strongly dependent on Yku80 (Hediger et al. 2002).

Given that the perinuclear anchorage of Tel6R is only partially sensitive to sir4 deletion, we next tested the effect of Mps3-N' expression in this background. There was no additive effect on telomere displacement over the sir4provoked loss of anchoring, arguing that the residual association of Tel6R in S-phase cells expressing the Mps3- $\mathrm{N}^{\prime}$ fragment does not act through Sir4. This is consistent with data from Bupp et al. (2007), and argues for a third G1-phase anchorage pathway for native telomeres that is
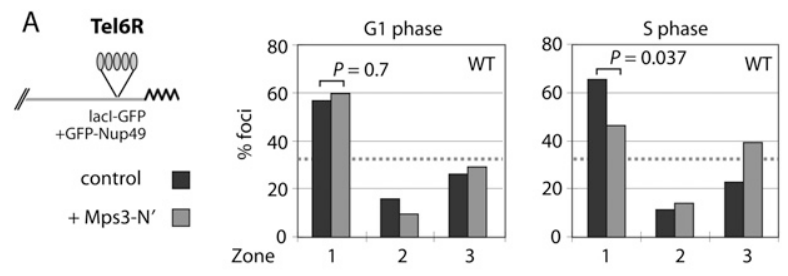

B Tel6R
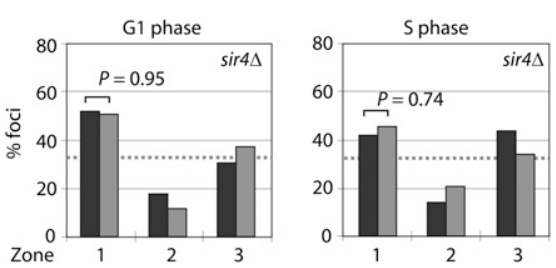

Figure 5. Peripheral nuclear localization of Tel6R depends on Mps3. (A) Localization of the lacO-tagged Tel6R (Hediger et al. 2002) at the NE was monitored in $3 \mathrm{D}$ through-focus stacks of images of live cells growing in agar. Cell cycle stages were determined by bud morphology. The strains used and cell numbers scored are as follows: GA-1459 (wild type, control; G1, $n=87$; S phase, $n=61$ ) compared with GA-1459 expressing Mps3-N' from a multicopy vector $(\mathrm{G} 1$ phase, $n=61$; $\mathrm{S}$ phase, $n=$ 56). (B) GA-1867 (sir4A, control; G1 phase, $n=62$; $\mathrm{S}$ phase, $n=$ 57) compared with GA-1867 expressing Mps3-N' from a multicopy vector (G1 phase, $n=51$; S phase, $n=53$ ). neither yKu- nor Sir4-dependent. While this was postulated previously in Taddei et al. (2004), the interpretation was complicated by the fact that Yku80 and Sir4 interact. Indeed, since sir4 or yku80 mutation could "neutralize" the function of the other, epistasis in native telomere anchoring is difficult to determine.

Nonetheless, since we showed that lexA-Est2 targets chromatin to the NE in an Mps3-dependent manner, we were interested to know whether Est2 tethering works in parallel to or in cooperation with the Sir4 anchoring pathway. To this end, we compared Tel6R anchoring in wild-type cells with its anchoring in cells deleted for sir4 alone or for both sir4 and est2. Telomere position was scored in the latter strain before cells entered senescence. Intriguingly, in G1-phase cells we see a strongly additive effect of sir4 and est2 deletions on telomere anchoring, while in S-phase cells the sir4 deletion led to strong delocalization alone, and there was no additional effect upon loss of est2 (Supplemental Fig. S3). These results indicate that in G1-phase cells Sir4 and Est2 act on distinct pathways, while at natural telomeres Sir4 may contribute to the yKu-telomerase anchorage in S phase. It is not clear whether sir4 deletion directly impairs the yKu-telomerase-Mps3 interaction in S phase, or alters telomere structure such that the interaction with Mps3 is impaired.

\section{Synthetic interactions between Mps3-N' and tells affect telomere stability}

The absence of the $S$. cerevisiae ATM homolog Tell leads to a stable but short telomere repeat length (Greenwell et al. 1995). This reduces the number of Rap1-binding sites per chromosome end, and in turn results in less SIR complex at telomeres. The reduced level of SIR complex at telomeres in tel1 cells correlates with a weakened telomere position effect (TPE) (data not shown), yet tel1 cells retain fairly robust telomere anchoring (Hediger et al. 2006). We speculate that telomere anchoring in tel1 cells may therefore be even more dependent on the interaction of Yku80, telomerase, and Mps3.

This reflection led us to test whether a perturbation of the yKu-telomerase-Mps3 pathway of anchoring affects telomere maintenance in tel1 mutant cells. We examined both telomere length and subtelomeric repeat stability in isogenic wild-type and tel1 deletion strains in which Mps3-N' was expressed to interfere with this telomere anchor. Indeed, whereas tell cells are not themselves senescent, the overexpression of Mps3- $\mathrm{N}^{\prime}$ led to a senescence-like phenotype, which limited proliferative capacity and resulted in an accumulation of divisionarrested, large-budded cells (Fig. 6A). This phenotype was not observed upon equally robust Mps3-N' expression in wild-type cells (see red fluorescence in Fig. 6A).

We quantified this perturbation by scoring unbudded (G1) and budded (S-G2) large budded/dumbbell-shaped cells (G2/M-arrested) in tel1 and tel1 + Mps3-N' cultures (Fig. 6B). Whereas roughly $60 \%$ of all tel1 cells bearing an empty vector were unbudded (G1-phase cells), only 20\% of all tel1 + Mps3-N' cells were in G1 phase (Fig. 6B). 
A

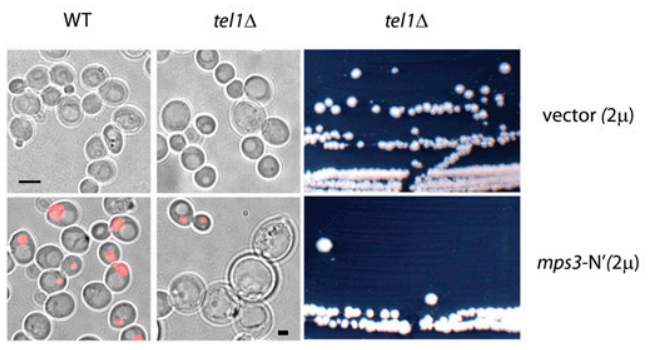

B

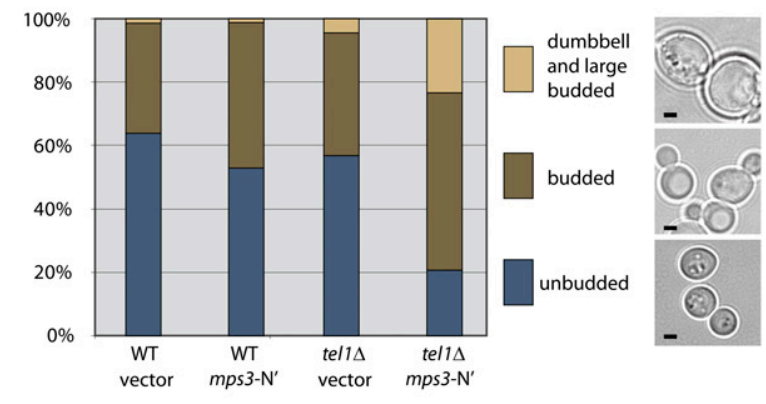

C

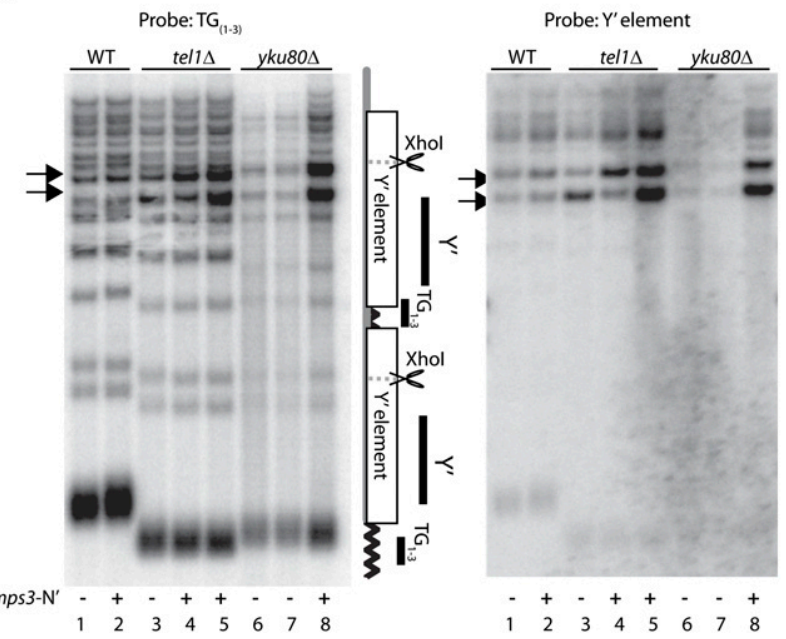

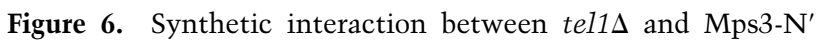
expression. (A) Transmission light images of wild-type yeast strain GA-426 (Tel5R-ADE2-TG) and GA-1370 (tel1s in GA-426) transformed with either empty vector $(2 \mu)$ (top panels) or with $2 \mu$ carrying Mps3-N' fused to mCherry (bottom panels). Diffuse nuclear fluorescence of mCherry (red) confirms Mps3- $\mathrm{N}^{\prime}$ expression. Bar, $5 \mu \mathrm{m}$. The right panels show a tel1s strain carrying vector only (top) or Mps3- $\mathrm{N}^{\prime}$ (bottom) on selective media. (B) Comparison of unbudded, budded, and large-budded dumbbell-shaped cells in wild-type (GA-426) and tel1s cells (GA-1370) cells transformed with either empty vector $(2 \mu)$ or $2 \mu$ bearing Mps3-N'. Numbers of cells scored for samples from left to right are 138, 270, 338, and 159. Phase images of each category are shown at right. Bar, $5 \mu \mathrm{m}$. $(C$, left $)$ Southern blot of Xhol-digested genomic DNA probed with TG repeats as described (Craven and Petes 1999). (Right) The same membrane was stripped and probed with $\mathrm{P}^{32}$-labeled $\mathrm{Y}^{\prime}$ DNA.

Instead, tel1 + Mps3- $\mathrm{N}^{\prime}$ cells accumulated as largebudded and dumbbell-shaped cells, consistent with an arrest in late G2/M (Fig. 6B). We were not able to detect modification of Rad53, which is indicative of checkpoint activation, although fragmented nuclei and anaphase bridges were detected at a higher frequency in the senescent population (data not shown).

To determine whether telomeres shortened continuously under these conditions, as in telomerase deficient mutants, we performed a Southern blot to monitor telomere length and/or possible rearrangements /Craven and Petes 1999). However, no change in the composition of $\mathrm{Y}^{\prime}$ DNA and no significant alteration of telomere length were observed when Mps3-N' was overexpressed in wildtype cells (Fig. 6C). Nonetheless, in cells lacking the Tel1mediated control over telomerase, we observed a significant amount of $\mathrm{Y}^{\prime}$ DNA amplification whenever cells overexpressed Mps3-N" (Fig. 6C, cf. lanes 3,5). In contrast, TG repeat length was not affected beyond the stable shortening that accompanies tel1 deletion. We interpret this as showing that the Mps3-mediated anchoring does not affect the elongation activity of telomerase per se, although Mps3- $\mathrm{N}^{\prime}$-induced telomere displacement significantly enhanced recombination between telomere ends. The amplification was specific for $\mathrm{Y}^{\prime}$ repeats, as telomereproximal genes showed no increase in copy number. Moreover, there was no detectable accumulation of extrachromosomal circles of $\mathrm{Y}^{\prime}$ repeats (data not shown).

We next asked whether this Mps3- $\mathrm{N}^{\prime}$-induced effect was dependent on $\mathrm{yKu}$ function, by scoring telomere length and $\mathrm{Y}^{\prime}$ amplification in a strain deleted for yku80. We noted a similar degree of $\mathrm{Y}^{\prime}$ amplification upon Mps3-N' overexpression in yku80 as in tel1 strains, arguing that Mps3- $\mathrm{N}^{\prime}$-induced uncapping and recombination does not require $\mathrm{yKu}$. This also argues that the protection from recombination that is disrupted by Mps3- $\mathrm{N}^{\prime}$ overexpression can be achieved through Yku80-mediated anchorage. We speculate that the recombination events provoked by Mps3-N' overexpression, which correlate with loss of anchoring, result from $\mathrm{S}$-phase replication, since Mps3- $\mathrm{N}^{\prime}$ overexpression does not release native telomeres in G1 phase cells (Fig. 5).

\section{Discussion}

Here we sought to understand the functional significance of telomere anchoring at the nuclear periphery in $S$. cerevisiae. Yeast is ideal for testing the functional compartmentation of the eukaryotic nucleus, as it provides a powerful system for the manipulation and monitoring of telomere position. Our analyses led to the discovery that budding yeast telomerase itself has an affinity for the NE. Telomerase forms part of a multiprotein pathway that is necessary for the perinuclear anchoring of telomeres during $S$ phase. We identified the evolutionarily conserved protein Mps3 as its putative peripheral anchor. Mps3 was shown previously to interact with Est1, an essential noncatalytic component of telomerase (Uetz et al. 2000; Antoniacci et al. 2007). It was proposed to anchor telomeres through Sir4 (Bupp et al. 2007). Besides its tethering function, Mps3 is involved in protecting telomeres from inappropriate recombination events. These were scored in strains that lack the ATM homolog Tell and express the $\mathrm{N}$ terminus of $\mathrm{Mps} 3$, which competes for 
the Est1-Mps3 interaction. Earlier work shows a correlation between loss of anchoring and telomere shortening in a tel1s background. Indeed, telomere delocalization correlates with repeat shortening in cells in which the viral transcriptional activator VP16, or the natural subtelomeric ligands Reb1 and Tbf1, are targeted to telomeres (Hediger et al. 2006). These previous experiments, however, did not directly implicate telomerase in the subnuclear positioning of chromatin.

The transcription of telomeric repeats also leads to shorter yeast telomeres, at least in a mutant that is unable to degrade nascent mRNAs (Luke et al. 2008). Whereas membrane anchorage per se does not silence subtelomeric genes (Mondoux et al. 2007), the clustering of telomeric repeats and sequestration of SIR factors does promote transcriptional repression (Andrulis et al. 1998; Taddei et al. 2009). Thus, an intact telomere that supports SIR-mediated silencing is likely to be anchored and nontranscribed, both of which are characteristics that stabilize telomerase binding and function. To this we add the finding that telomerase itself helps anchor telomeres during S phase, a time at which the interaction of SIR factors with nucleosomes is likely to be disrupted by the passing replication fork. This would explain why the presence of a second, silencing-independent pathway for telomere anchorage is physiologically relevant.

\section{Relevance of position for telomere stability}

Although the precise subnuclear positioning of telomeres differs in yeast and mammalian cells, telomere localization has been shown to contribute to the processing of telomerase components and the regulation of telomerase activity in mammalian cells as well (Wong et al. 2002; Tomlinson et al. 2006; Cristofari et al. 2007). Indeed, a component of Cajal bodies appears to be an essential cofactor of mammalian telomerase (Venteicher et al. 2009). Consistent with the notion that telomerase assembles on telomeres that are tethered, de Lange and colleagues (van Steensel et al. 1998) have monitored increased end-to-end fusion events in cells that are depleted for the telomere binding protein TRF2. TRF2 depletion also correlates with enhanced telomere mobility that seems to allow chromosome ends to explore larger subnuclear territories by stochastic diffusion (Dimitrova et al. 2008). Similarly, we monitored enhanced recombination events in yeast when telomeres lose Mps3-mediated anchoring, at least in tel1-deficient strains.

The data we presented here are consistent with the hypothesis that subnuclear localization contributes to an "open" or "closed" state of telomeres, which is thought to correlate with altered states of accessibility for telomerase (Blackburn 2001; Teixeira et al. 2004). The open state may favor telomerase loading, as opposed to shunting the damage to another repair mechanism, be it end-joining or homologous recombination (HR). It is noteworthy that a spatial mapping of Rad52 foci in relation to the NE suggested that HR events occur preferentially in the nuclear lumen; Rad52 foci were strongly depleted from the nuclear periphery (Bystricky et al. 2009). This was true whether the source of damage was an enzymatically induced DNA double-strand break (DSB), alkylation by MMS, or spontaneous replication fork collapse. Given this internal localization for $\mathrm{HR}$, it is easy to imagine that sequestration at the NE disfavors recombination events. This is supported not only by the amplification of $\mathrm{Y}^{\prime}$ elements that is detected when S-phase-specific anchorage is disrupted (Fig. 6), but also by the doubling in the rate of direct repeat recombination of an internal LEU2 reporter in a strain bearing the mps3 $475-150$ deletion (J. Gardner and S. Jasperson, pers. comm.).

Consistently, it was reported recently that recombination among yeast rDNA repeats is suppressed by tethering the rDNA at the NE by Heh1, a protein related to Man1, a mammalian component of the inner NE (also called Src1) (Mekhail et al. 2008). Loss of Heh1 caused decondensation of the rDNA, partial release of the nucleolus from the nuclear periphery, and increased recombination (Mekhail et al. 2008). Although the Heh1 suppression of rDNA recombination is most likely unlinked to the effect of Mps3 anchoring on telomeres, these results reinforce the notion that NE tethering is able to repress recombination events independently of the transcriptional status of the locus.

We suggest that telomere anchoring by Mps3 at the NE protects chromosomal ends from inappropriate repair by nonreciprocal recombination events, which most likely arise during replication. Interference with Mps3mediated telomere anchoring in a sensitized background like tel1 led to $\mathrm{Y}^{\prime}$ amplification and a senescence-like growth arrest. Amplification appears to occur primarily at telomeric elements, as subtelomeric genes did not change copy number (data not shown). We detected DNA bridges between daughter nuclei in late anaphase cells of Mps3-N'-overexpressing cells, yet these phenotypes were not detected unless telomere capping was compromised by tel1 deletion (Fig. 6). Consistent with these observations, however, yeast deleted for this intranuclear domain of Mps3 showed increased ectopic recombination after induction of a DSB (Oza et al. 2009). There is accumulating evidence that telomere-specific factors can be recruited to DSBs along with repair factors like the Mre11-Rad50-Xrs2 complex (Trujillo et al. 2003; Oza et al. 2009). Surprisingly, recombination factors can also be found at telomeres. Thus, a delicate balance is maintained between recombination-mediated repair and telomerase loading, which itself appears to suppress recombination. Whether telomerase-mediated anchoring retards resection or strand invasion is at present unclear. Intriguingly, Ku and Mre11 act not only as repair proteins, but also as positive regulators of telomere length (for review, see Rog and Cooper 2008). Thus, in this context, it is plausible that the subnuclear position of DNA will help regulate the outcome of repair protein association to chromosomal ends, be they telomeres or DSBs.

\section{The yKu-telomerase-Mps3 anchorage complex}

Our search for a perinuclear anchor for yKu revealed not only that Yku80-mediated anchoring requires Tlc1 in 
$S$ phase, but also that the catalytic subunit of telomerase Est2 can relocate chromatin to the NE. Both events required the interaction of Est1 with the nucleoplasmic domain of the inner NE protein, Mps3. This novel function for telomerase reinforces the link between nuclear localization and telomere maintenance. Telomerase helps Yku80 anchor chromatin in S phase, which is precisely when Est1 is expressed and Est2 recruitment to telomeres peaks (Taggart et al. 2002; Schramke et al. 2004). Indeed, the cell cycle regulation of this anchoring pathway (Taddei et al. 2004) may reflect the fact that Yku80 is found in different complexes, enabling it to fulfill different roles in repair, transcriptional silencing, and telomere protection.

Since Sir4 also was shown to interact with yKu and $\mathrm{Mps} 3$, and to contribute to telomere anchoring in S phase (Taddei et al. 2004; Bupp et al. 2007), we cannot exclude that a single complex of Sir4, Yku70/Yku80, Tlc1, Est2, and Est1 anchors telomeres to the evolutionarily conserved SUN domain protein Mps3. Importantly, interfering with this pathway perturbed telomere position in S-phase cells and enhanced the frequency of telomeric recombination events.

The pathway of perinuclear anchoring through Yku80telomerase and Mps3 is distinct from the recently reported relocation of irreparable breaks to the nuclear pore subcomplex Nup84/Slx5/Slx8 (Nagai et al. 2008). Indeed, the binding of lexA-yku80-4 or lexA-Est2 to chromatin does not promote significant colocalization with the NPC, while the targeting of lexA-Nup84 does. We further note that the outcomes of the two peripheral tethering events are opposite: Mps3 binding suppresses recombination, while the Nup84/Slx5/Slx8 interaction facilitates an alternative pathway of repair that enhances gene conversion, possibly through template switch recombination (Nagai et al. 2008).

It is noteworthy that the human homolog of Slx $5 / S 1 x 8$, RNF4, is involved in the degradation of SUMO-modified PML protein (Lallemand-Breitenbach et al. 2008; Tatham et al. 2008; Weisshaar et al. 2008). Intriguingly, PML bodies are implicated in the alternative lengthening of telomeres through recombination, the so-called ALT pathway (Bernardi and Pandolfi 2007). By analogy, we speculate that the loss of Mps3-telomerase interaction may lead to the transfer of telomeric chromatin to the Nup84/Slx5/Slx8 complex, where it may be processed for recombinational repair. Indeed, in the absence telomerase activity, the Slx5-Slx8 complex in budding yeast appears to facilitate the formation or growth of type II survivors, in which telomere sequences (TG repeats) themselves are amplified by recombination events that require Rad52 and Sgs1 (Azam et al. 2006; McEachern and Haber 2006). Thus, delivery to the Nup84/Slx5-Slx8 complex appears to be involved in the final choice of recombinational repair pathways (Nagai et al. 2008), and we propose that this transfer may also happen for DSBs that are first recruited to Mps3 (Oza et al. 2009). Further analysis is needed to determine how these distinct perinuclear anchorage sites orchestrate repair, and whether one or both also involves the G1-phase pathway that implicates the RFC-associated factor Ctf18 in tethering (Hiraga et al. 2006). Our study makes it clear that telomerase itself is likely to serve as part of a cell cycle-specific anchorage complex for yeast telomeres, on a pathway that seems to be intimately involved in protecting telomere ends from recombination.

\section{Materials and methods}

Yeast techniques and live fluorescence microscopy

For live imaging, cultures were grown in synthetic medium to a concentration of $<1 \times 10^{5}$ cells per milliliter. Microscopy was performed at $30^{\circ} \mathrm{C}$ on cells spread on agarose patches containing synthetic complete medium with $4 \%$ glucose. Images for zoning measurements were captured on a Metamorph-driven Olympus IX70 widefield microscope equipped with a Coolsnap HQ camera from Roper Scientific Photometrics. Cells expressing GFPNUP49 and lacI-GFP bound to lacO were imaged by acquiring stacks with a step size of $0.2 \mu \mathrm{m}$ using a wavelength of $475 \mathrm{~nm}$ for excitation. For cells bearing the nup133 N-terminal deletion (amino acids 44-246) (Doye et al. 1994) CFP-Nup49 was used to identify pore clusters and lacO/GFP-lacI was used to monitor the targeted locus. Stacks of 21 images with a step size of $0.2 \mu \mathrm{m}$ were acquired, with the wavelength alternated between $437 \mathrm{~nm}$ (CFP) and $517 \mathrm{~nm}$ (YFP) once per plane. A 100×/1.4 Oil PlanApochromat objective from Zeiss was used. Deconvolution was performed using the Huygens software (calculated PSF) and Imaris (Biplane) Software for analysis of colocalization. The Fisher's Exact Test for Count Data was performed using the R-project software package (http://www.r-project.org). Plasmids and yeast strains used in this study are described in the Supplemental Material. Deletion and modification of genes were achieved by plasmid-borne or PCR-based methods (Longtine et al. 1998), and were verified by PCR and phenotypic analysis. Telomeric positioning data were analyzed as described (Hediger et al. 2004; Taddei et al. 2004).

\section{Acknowledgments}

We thank the FMI imaging platform for help and advice. This research was supported by the Novartis Research foundation. H.S. was supported by the "Frontiers in Genetics" NCCR program (sponsored by the Swiss National Science Foundation), and H.F. was supported by a Marie Curie Fellowship.

\section{References}

Andrulis, E.D., Neiman, A.M., Zappulla, D.C., and Sternglanz, R. 1998. Perinuclear localization of chromatin facilitates transcriptional silencing. Nature 394: 592-595.

Andrulis, E.D., Zappulla, D.C., Ansari, A., Perrod, S., Laiosa, C.V., Gartenberg, M.R., and Sternglanz, R. 2002. Esc1, a nuclear periphery protein required for Sir4-based plasmid anchoring and partitioning. Mol. Cell. Biol. 22: 8292-8301.

Antoniacci, L.M., Kenna, M.A., and Skibbens, R.V. 2007. The nuclear envelope and spindle pole body-associated Mps3 protein bind telomere regulators and function in telomere clustering. Cell Cycle 6: 75-79.

Azam, M., Lee, J.Y., Abraham, V., Chanoux, R., Schoenly, K.A., and Johnson, F.B. 2006. Evidence that the S. cerevisiae Sgs1 protein facilitates recombinational repair of telomeres during senescence. Nucleic Acids Res. 34: 506-516.

Bernardi, R. and Pandolfi, P.P. 2007. Structure, dynamics and functions of promyelocytic leukaemia nuclear bodies. Nat. Rev. Mol. Cell Biol. 8: 1006-1016. 
Berthiau, A.S., Yankulov, K., Bah, A., Revardel, E., Luciano, P., Wellinger, R.J., Geli, V., and Gilson, E. 2006. Subtelomeric proteins negatively regulate telomere elongation in budding yeast. $E M B O$ J. 25: 846-856.

Blackburn, E.H. 2001. Switching and signaling at the telomere. Cell 106: 661-673.

Bupp, J.M., Martin, A.E., Stensrud, E.S., and Jaspersen, S.L. 2007. Telomere anchoring at the nuclear periphery requires the budding yeast Sad1-UNC-84 domain protein Mps3. J. Cell Biol. 179: 845-854.

Bystricky, K., Van Attikum, H., Montiel, M.D., Dion, V., Gehlen, L., and Gasser, S.M. 2009. Regulation of nuclear positioning and dynamics of the silent mating type loci by the yeast Ku70/Ku80 complex. Mol. Cell. Biol. 29: 835-848.

Chikashige, Y., Tsutsumi, C., Yamane, M., Okamasa, K., Haraguchi, T., and Hiraoka, Y. 2006. Meiotic proteins bqt 1 and bqt 2 tether telomeres to form the bouquet arrangement of chromosomes. Cell 125: 59-69.

Conrad, M.N., Lee, C.Y., Wilkerson, J.L., and Dresser, M.E. 2007. MPS3 mediates meiotic bouquet formation in Saccharomyces cerevisiae. Proc. Natl. Acad. Sci. 104: 8863-8868.

Craven, R.J. and Petes, T.D. 1999. Dependence of the regulation of telomere length on the type of subtelomeric repeat in the yeast Saccharomyces cerevisiae. Genetics 152: 1531-1541.

Cristofari, G., Adolf, E., Reichenbach, P., Sikora, K., Terns, R.M., Terns, M.P., and Lingner, J. 2007. Human telomerase RNA accumulation in Cajal bodies facilitates telomerase recruitment to telomeres and telomere elongation. Mol. Cell 27: 882-889.

Dimitrova, N., Chen, Y.C., Spector, D.L., and de Lange, T. 2008. 53BP1 promotes non-homologous end joining of telomeres by increasing chromatin mobility. Nature 456: 524-528.

Doye, V., Wepf, R., and Hurt, E.C. 1994. A novel nuclear pore protein Nup133p with distinct roles in poly(A)+ RNA transport and nuclear pore distribution. EMBO J. 13: 6062-6075.

Gartenberg, M.R., Neumann, F.R., Laroche, T., Blaszczyk, M., and Gasser, S.M. 2004. Sir-mediated repression can occur independently of chromosomal and subnuclear contexts. Cell 119: 955-967.

Greenwell, P.W., Kronmal, S.L., Porter, S.E., Gassenhuber, J., Obermaier, B., and Petes, T.D. 1995. TEL1, a gene involved in controlling telomere length in S. cerevisiae, is homologous to the human ataxia telangiectasia gene. Cell 82: 823-829.

Hediger, F., Neumann, F.R., Van Houwe, G., Dubrana, K., and Gasser, S.M. 2002. Live imaging of telomeres: $y K u$ and Sir proteins define redundant telomere-anchoring pathways in yeast. Curr. Biol. 12: 2076-2089.

Hediger, F., Taddei, A., Neumann, F.R., and Gasser, S.M. 2004. Methods for visualizing chromatin dynamics in living yeast. Methods Enzymol. 375: 345-365.

Hediger, F., Berthiau, A.S., van Houwe, G., Gilson, E., and Gasser, S.M. 2006. Subtelomeric factors antagonize telomere anchoring and Tell-independent telomere length regulation. EMBO J. 25: 857-867.

Hiraga, S., Robertson, E.D., and Donaldson, A.D. 2006. The Ctf18 RFC-like complex positions yeast telomeres but does not specify their replication time. EMBO J. 25: 1505-1514.

Jady, B.E., Richard, P., Bertrand, E., and Kiss, T. 2006. Cell cycledependent recruitment of telomerase RNA and Cajal bodies to human telomeres. Mol. Biol. Cell 17: 944-954.

Jaspersen, S.L., Giddings Jr., T.H., and Winey, M. 2002. Mps3p is a novel component of the yeast spindle pole body that interacts with the yeast centrin homologue Cdc31p. J. Cell Biol. 159: 945-956.

Lallemand-Breitenbach, V., Jeanne, M., Benhenda, S., Nasr, R., Lei, M., Peres, L., Zhou, J., Zhu, J., Raught, B., and de The, H.
2008. Arsenic degrades PML or PML-RAR $\alpha$ through a SUMO-triggered RNF4/ubiquitin-mediated pathway. Nat. Cell Biol. 10: 547-555.

Larose, S., Laterreur, N., Ghazal, G., Gagnon, J., Wellinger, R.J., and Elela, S.A. 2007. RNase III-dependent regulation of yeast telomerase. J. Biol. Chem. 282: 4373-4381.

Lingner, J., Cooper, J.P., and Cech, T.R. 1995. Telomerase and DNA end replication: No longer a lagging strand problem? Science 269: 1533-1534.

Loeillet, S., Palancade, B., Cartron, M., Thierry, A., Richard, G.F., Dujon, B., Doye, V., and Nicolas, A. 2005. Genetic network interactions among replication, repair and nuclear pore deficiencies in yeast. DNA Repair (Amst.) 4: 459-468.

Longtine, M.S., McKenzie III, A., Demarini, D.J., Shah, N.G., Wach, A., Brachat, A., Philippsen, P., and Pringle, J.R. 1998. Additional modules for versatile and economical PCR-based gene deletion and modification in Saccharomyces cerevisiae. Yeast 14: 953-961.

Luke, B., Panza, A., Redon, S., Iglesias, N., Li, Z., and Lingner, J. 2008. The Ratlp 5' to $3^{\prime}$ exonuclease degrades telomeric repeat-containing RNA and promotes telomere elongation in Saccharomyces cerevisiae. Mol. Cell 32: 465-477.

McEachern, M.J. and Haber, J.E. 2006. Break-induced replication and recombinational telomere elongation in yeast. Annu. Rev. Biochem. 75: 111-135.

Mekhail, K., Seebacher, J., Gygi, S.P., and Moazed, D. 2008. Role for perinuclear chromosome tethering in maintenance of genome stability. Nature 456: 667-670.

Mondoux, M.A., Scaife, J.G., and Zakian, V.A. 2007. Differential nuclear localization does not determine the silencing status of Saccharomyces cerevisiae telomeres. Genetics 177: 20192029.

Nagai, S., Dubrana, K., Tsai-Pflugfelder, M., Davidson, M.B., Roberts, T.M., Brown, G.W., Varela, E., Hediger, F., Gasser, S.M., and Krogan, N.J. 2008. Functional targeting of DNA damage to a nuclear pore-associated SUMO-dependent ubiquitin ligase. Science 322: 597-602.

Nishikawa, S., Terazawa, Y., Nakayama, T., Hirata, A., Makio, T., and Endo, T. 2003. Nep98p is a component of the yeast spindle pole body and essential for nuclear division and fusion. J. Biol. Chem. 278: 9938-9943.

Oza, P., Jaspersen, S.L., Miele, A., Dekker, J., and Peterson, C.L. 2009. Mechanisms that regulate localization of a DNA double-strand break to the nuclear periphery. Gene \& Dev. (this issue). doi: 10.1101/gad.1782209.

Palancade, B., Liu, X., Garcia-Rubio, M., Aguilera, A., Zhao, X., and Doye, V. 2007. Nucleoporins prevent DNA damage accumulation by modulating Ulp1-dependent sumoylation processes. Mol. Biol. Cell 18: 2912-2923.

Rog, O. and Cooper, J.P. 2008. Telomeres in drag: Dressing as DNA damage to engage telomerase. Curr. Opin. Genet. Dev. 18: 212-220.

Roy, R., Meier, B., McAinsh, A.D., Feldmann, H.M., and Jackson, S.P. 2004. Separation-of-function mutants of yeast Ku80 reveal a Yku80p-Sir4p interaction involved in telomeric silencing. J. Biol. Chem. 279: 86-94.

Schramke, V., Luciano, P., Brevet, V., Guillot, S., Corda, Y., Longhese, M.P., Gilson, E., and Geli, V. 2004. RPA regulates telomerase action by providing Est $1 \mathrm{p}$ access to chromosome ends. Nat. Genet. 36: 46-54.

Sexton, T., Schober, H., Fraser, P., and Gasser, S.M. 2007. Gene regulation through nuclear organization. Nat. Struct. Mol. Biol. 14: 1049-1055.

Shaner, N.C., Campbell, R.E., Steinbach, P.A., Giepmans, B.N., Palmer, A.E., and Tsien, R.Y. 2004. Improved monomeric red, orange and yellow fluorescent proteins derived from 
Schober et al.

Discosoma sp. red fluorescent protein. Nat. Biotechnol. 22: $1567-1572$.

Stellwagen, A.E., Haimberger, Z.W., Veatch, J.R., and Gottschling, D.E. 2003. Ku interacts with telomerase RNA to promote telomere addition at native and broken chromosome ends. Genes \& Dev. 17: 2384-2395.

Taddei, A., Hediger, F., Neumann, F.R., Bauer, C., and Gasser, S.M. 2004. Separation of silencing from perinuclear anchoring functions in yeast Ku80, Sir4 and Esc1 proteins. EMBO J. 23: 1301-1312.

Taddei, A., Van Houwe, G., Nagai, S., Erb, I., van Nimwegen, E.J., and Gasser, S.M. 2009. The functional importance of telomere clustering: Global changes in gene expression result from SIR factor dispersion. Genome Res. doi: 10.1101/gr. 083881.108 .

Taggart, A.K., Teng, S.C., and Zakian, V.A. 2002. Estlp as a cell cycle-regulated activator of telomere-bound telomerase. Science 297: 1023-1026.

Tatham, M.H., Geoffroy, M.C., Shen, L., Plechanovova, A., Hattersley, N., Jaffray, E.G., Palvimo, J.J., and Hay, R.T. 2008. RNF4 is a poly-SUMO-specific E3 ubiquitin ligase required for arsenic-induced PML degradation. Nat. Cell Biol. 10: $538-546$.

Teixeira, M.T., Arneric, M., Sperisen, P., and Lingner, J. 2004. Telomere length homeostasis is achieved via a switch between telomerase-extendible and -nonextendible states. Cell 117: 323-335.

Ting, N.S., Yu, Y., Pohorelic, B., Lees-Miller, S.P., and Beattie, T.L. 2005. Human Ku70/80 interacts directly with hTR, the RNA component of human telomerase. Nucleic Acids Res. 33: 2090-2098.

Tomlinson, R.L., Ziegler, T.D., Supakorndej, T., Terns, R.M., and Terns, M.P. 2006. Cell cycle-regulated trafficking of human telomerase to telomeres. Mol. Biol. Cell 17: 955-965.

Trujillo, K.M., Roh, D.H., Chen, L., Van Komen, S., Tomkinson, A., and Sung, P. 2003. Yeast xrs2 binds DNA and helps target rad50 and mre11 to DNA ends. J. Biol. Chem. 278: 4895748964.

Tzur, Y.B., Wilson, K.L., and Gruenbaum, Y. 2006. SUN-domain proteins: 'Velcro' that links the nucleoskeleton to the cytoskeleton. Nat. Rev. Mol. Cell Biol. 7: 782-788.

Uetz, P., Giot, L., Cagney, G., Mansfield, T.A., Judson, R.S., Knight, J.R., Lockshon, D., Narayan, V., Srinivasan, M., Pochart, P., et al. 2000. A comprehensive analysis of proteinprotein interactions in Saccharomyces cerevisiae. Nature 403: 623-627.

van Steensel, B., Smogorzewska, A., and de Lange, T. 1998. TRF2 protects human telomeres from end-to-end fusions. Cell 92: 401-413.

Venteicher, A.S., Abreu, E.B., Meng, Z., McCann, K.E., Terns, R.M., Veenstra, T.D., Terns, M.P., and Artandi, S.E. 2009. A human telomerase holoenzyme protein required for Cajal body localization and telomere synthesis. Science 323: 644648.

Weisshaar, S.R., Keusekotten, K., Krause, A., Horst, C., Springer, H.M., Gottsche, K., Dohmen, R.J., and Praefcke, G.J. 2008. Arsenic trioxide stimulates SUMO-2/3 modification leading to RNF4-dependent proteolytic targeting of PML. FEBS Lett. 582: $3174-3178$.

Wong, J.M., Kusdra, L., and Collins, K. 2002. Subnuclear shuttling of human telomerase induced by transformation and DNA damage. Nat. Cell Biol. 4: 731-736.

Zhao, X., Wu, C.Y., and Blobel, G. 2004. Mlp-dependent anchorage and stabilization of a desumoylating enzyme is required to prevent clonal lethality. J. Cell Biol. 167: 605-611. 


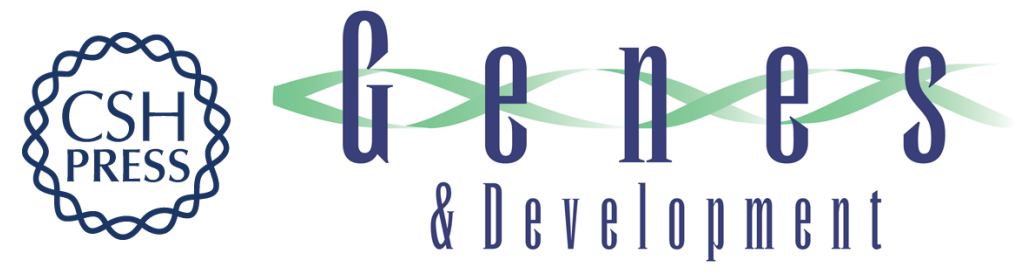

\section{Yeast telomerase and the SUN domain protein Mps3 anchor telomeres and repress subtelomeric recombination}

Heiko Schober, Helder Ferreira, Véronique Kalck, et al.

Genes Dev. 2009, 23:

Access the most recent version at doi:10.1101/gad.1787509

\section{Supplemental http://genesdev.cshlp.org/content/suppl/2009/04/16/23.8.928.DC1 \\ Material}

Related Content Life on the edge: telomeres and persistent DNA breaks converge at the nuclear periphery

Marc R. Gartenberg

Genes Dev. May , 2009 23: 1027-1031

References This article cites 57 articles, 23 of which can be accessed free at: http://genesdev.cshlp.org/content/23/8/928.full.html\#ref-list-1

Articles cited in:

http://genesdev.cshlp.org/content/23/8/928.full.html\#related-urls

\section{License}

Email Alerting

Service

Receive free email alerts when new articles cite this article - sign up in the box at the top right corner of the article or click here.

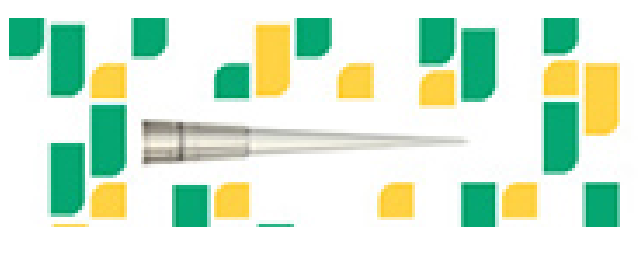

Focused on your science. 